\title{
The Effect of Topological Spaces and fg- Closed Sets on The Types of Digital Images
}

\author{
Alyaa Yousif Khudayir ${ }^{1, *}$ and Hind Rustum Mohammed ${ }^{2, *}$ \\ ${ }^{1,2}$ Department of mathematics, Faculty of Computer Science and mathematics, University of Kufa, Iraq \\ ${ }^{{ }_{1} 1}$ Corresponding author: Alyaa Yousif Khudayir ${ }^{1}$, alyaay.khudhair@uokufa.edu.iq. \\ ${ }^{* 2}$ Corresponding author: Hind Rustum Mohammed², hindrustum.shaaban@uokufa.edu.iq
}

\begin{abstract}
There is a growing interest in studying and improving the characteristics of images and objects in the e-commerce environment. Digital topology is concerned with dealing with the properties and features of two-dimensional (2D) or three-dimensional (3D) digital images such as borders, shapes, the intensity of illumination, and other characteristics. This paper aims to introduce and study new classes of fg-disconnected space and compactly fg-closed set, which could impact the brightness and brightness of the internal components of the types of color images, gray and binary. The paper also aims to find the effect of implementing fg-disconnected space and compactly fg-closed set to determine the brightness and brightness of the internal components of the types of color images, gray and binary. Each research plate contains 30 images of each type of image. Ten different images were chosen at the same time to be analyzed and executed using the proposed system based on MATLAB software. The study proved that higher brightness and light will disappear and delete the components of the image of any kind. This aimed to make the image white and opposite color, the greater darkness, and luminescence will make the picture color mysterious and turn to black.
\end{abstract}

Keywords: fg- continuous function; fg-disconnected space; compactly- fg-closed set; image processing; image enhancement. 


\section{Introduction}

There is an increasing interest in analyzing and enhancing the characteristics of images and objects and their features in the e-commerce environment. Digital topology deals with the study of the properties and features of twodimensional or three-dimensional digital objects such as borders, shapes, the intensity of illumination, and other characteristics (Al-Omeri \& Jafari, 2019). Image enhancement has become a need for many applications to improve the quality and content of original images to provide 'better' input to other automated image processing techniques (Al-Hatmi \& Yousif, 2017; Hasoon et al., 2011). Image enhancement aims to improve the digital image to be more convenient for viewing and analysis. Improvements include removing noise, sharpening or brightening the image, making it easier to identify key features (Shaaban, 2021; Hassin \& Abbood, 2021).

In 1970, Levine. $\mathrm{N}$ initiated definition of generalized closed, a subset B of space $\mathrm{X}$ is called generalized closed ( $\mathrm{g}$ closed) if ${ }^{-} \mathrm{B} \subseteq \mathrm{Z}$ whenever $\mathrm{B} \subseteq \mathrm{Z}$ and $\mathrm{Z}$ is open set (Levine N.,1970). Navalagi, G.B, introduces the concept feebly open set-in topology (Navalagi, 2000). The work suggested by Resan includes the definition of feebly- generalized closed (fg-closed), a subset $B$ of $X$ is called feebly- generalized closed (Resan D., 2011). If ${ }^{-} \mathrm{B}^{\wedge} \mathrm{f} \subseteq \mathrm{Z}$ whenever $\mathrm{B} \subseteq \mathrm{Z}$ and $\mathrm{Z}$ is $\mathrm{f}$-open set and generalized feebly- closed (gf-closed), If ${ }^{-} \mathrm{B}^{\wedge} \mathrm{f} \subseteq \mathrm{Z}$ whenever $\mathrm{B} \subseteq \mathrm{Z}$ and $\mathrm{Z}$ is open set (Ibraheem D., 2008).

This research explained the definition of fg-disconnected space and compactly fg-closed set. Also, we proved the obtained results based on the experimental methodology (Banakh \& Ravsky, 2020). However, the proposed method is described in section 3; we debate and appraise the experiment results in section 4 and the conclusion in Section 5.

\section{Theorems and Definitions}

This section introduces the concepts fg-disconnected space as well as compactly-fg-closed set and their properties (Banakh \& Ravsky, 2020).

\section{Definition (2.1) (Navalagi, 2000; Levine N.,1963).}

Let $(\mathrm{X}, \mathcal{T})$ be topological space. A subset $\mathrm{B}$ of the space $\mathrm{X}$ is called:

- semi-open, if $\mathrm{B} \subseteq \overline{\mathrm{B}}^{\circ}$ and semi-closed, if $\mathrm{B}^{\circ} \subseteq \mathrm{B}$.

- feebly- open, if $\mathrm{B} \subseteq{\overline{\mathrm{B}^{\circ}}}^{\circ}$ and $\mathrm{f}-$ closed, if $\overline{\overline{\mathrm{B}}}^{\circ} \subseteq \mathrm{B}$. 
Definition (2.2) (Caldas et al., 2003).

Let $\mathrm{X}$ a space and $\mathrm{A} \subseteq \mathrm{X}$. The intersection of all f-closed sets containing $\mathrm{A}_{\mathrm{i}}$ is said to be the $\mathrm{f}$ - closed of $\ddot{\mathrm{A}}$ and denoted by $\overline{\mathrm{A}}^{\mathrm{f}}$.

Remark (2.3) (Navalagi, 2000)

- Each open set is f-open set.

- Each closed set is f-closed set.

Definition (2.4) (Levine N.,1970).

A subset $\mathrm{B}$ of a space $\mathrm{X}$ is called generalized closed (g-closed) if $\overline{\mathrm{B}} \subseteq \mathrm{Z}$ whenever $\mathrm{B} \subseteq \mathrm{Z}$ and $\mathrm{Z}$ is open set. The complement of g-closed set is g- open set.

Definition (2.5) (Resan D., 2011; Ibraheem D., 2008).

If $(\mathrm{X}, \mathcal{T})$ is topological space and $B \subseteq \mathrm{X}$ then $\mathrm{B}$ is said to be:

- $\quad$ - generalized closed (fg-closed), If $\overline{\mathrm{B}}^{\mathrm{f}} \subseteq \mathrm{Z}$ whenever $\mathrm{B} \subseteq \mathrm{Z}$ and $\mathrm{Z}$ is f-open set.

- $\quad$ Generalized f- closed (gf-closed), If $\overline{\mathrm{B}}^{\mathrm{f}} \subseteq \mathrm{Z}$ whenever $\mathrm{B} \subseteq \mathrm{Z}$ and $\mathrm{Z}$ is open set.

The complement of fg-closed resp (gf- closed) in space X are fg-open resp (gf-open) set in X.

Example (2.6)

Suppose that $X=\{a ́, b, c ̧, u ̛ ́\}$ and $\mathcal{T}=\{\varnothing, X,\{b, c ̧\}\}$ be topological space on $X$ and let $H=\{a ́, c ̧$, ư $\}$ and $\mathrm{Z}=\{$ á $\}$ be sets, thus $\mathrm{H}$ and $\mathrm{Z}$ are fg-closed set (resp gf- closed) sets in $\mathrm{X}$.

Proposition (2.7)

- Every closed set is fg-closed set

- Every f-closed set is fg-closed set 


\section{Proof}

i. Suppose that $\mathrm{B}$ is closed set and $\mathrm{B} \subseteq \mathrm{Z}$ such that $\mathrm{Z}$ is $\mathrm{f}$-open set, then by Remark (2.3), $\mathrm{B}$ is f-closed set, $\mathrm{B}=$ $\overline{\mathrm{B}}^{\mathrm{f}}$, thus $\overline{\mathrm{B}}^{\mathrm{f}} \subseteq \mathrm{Z}$, then $\mathrm{B}$ is fg-closed set.

ii. Let $\mathrm{B}$ is $\mathrm{f}$-closed set and $\mathrm{B} \subseteq \mathrm{Z}$ such that $\mathrm{Z}$ is $\mathrm{f}$-open set, then $\mathrm{B}=\overline{\mathrm{B}}^{\mathrm{f}}$, therefore $\overline{\mathrm{B}}^{\mathrm{f}} \subseteq \mathrm{Z}$, thus $\mathrm{B}$ is fg-closed set.

The convers of $\mathrm{i}$, ii in proposition (2.7) are not true in general as the shown in following example.

\section{Example (2.8)}

Suppose that $X=\{a, b, c\}$ be a set and, $\mathcal{T}=\{\varnothing X,\{a\}\}$ be topological space on $X$. Notice that $C=\{b\}$ is fgclosed set but $\mathrm{C}$ is not closed in $\mathrm{X}$.

\section{Definition (2.9)}

A function $\dot{\mathrm{f}}:(\mathrm{X}, \tau) \rightarrow(\mathrm{Y}, \beta)$ is said to be a fg-continuous if $\mathrm{f}^{-1}(M)$ is fg-closed set in $\mathrm{X}$ for each $\mathrm{M}$ is fg-closed set in Y.

Clearly it is proved that a function $\dot{\mathrm{f}}:(\mathrm{X}, \mathcal{T}) \rightarrow(\mathrm{Y}, \beta)$ is said to be fg-continuous if and only if $\dot{\mathrm{f}}^{-1}(M)$ is fg- open set in for each $\mathrm{M}$ is fg-closed set in $\mathrm{Y}$.

\section{Definition (2.10)}

A space $(\mathrm{X}, \mathcal{T})$ is called fg-disconnected space if $\mathrm{X}$ can be written as a union of two non-empty disjoint fgopen set. But $(\mathrm{X}, \mathcal{T})$ is called fg-connected space if it is not fg-disconnected.

\section{Proposition (2.11)}

Every disconnected space is fg-disconnected space.

\section{Proof}

Let $(\mathrm{X}, \mathcal{T})$ is disconnected space, then there exist $\mathrm{C}, \mathrm{D}$ are open set such that $\mathrm{C} \neq \varnothing, \mathrm{D} \neq \varnothing$ and $\mathrm{C} \cap \mathrm{D}=$ $\varnothing$ and $C \cup D=X$. Since $C$ and $D$ are open set then by proposition (2. 7), C and D are fg-open sets, thus (X, $\mathcal{T}$ ) fg-disconnected space. 
The convers of proposition (2.11) is not true in general as the presented in following example.

Example (2. 12):

Let $X=\left\{\begin{array}{c}{\left[\begin{array}{ccc}10 & 3 & 52 \\ 5 & 2 & 70 \\ 18 & 1 & 30\end{array}\right],\left[\begin{array}{ccc}1 & 7 & 43 \\ 73 & 65 & 24 \\ 4 & 95 & 25\end{array}\right]} \\ {\left[\begin{array}{rrr}10 & 55 & 105 \\ 20 & 95 & 120 \\ 35 & 45 & 70\end{array}\right]}\end{array}\right\}$

be set and let $\mathcal{T}=\{\varnothing, \mathrm{X}\}$ be indiscrete space.

There are fg- open sets: $\emptyset, X,\left\{\left[\begin{array}{ccc}10 & 3 & 52 \\ 5 & 2 & 70 \\ 18 & 1 & 30\end{array}\right],\left[\begin{array}{ccc}1 & 7 & 43 \\ 73 & 65 & 24 \\ 4 & 95 & 25\end{array}\right]\right\}$

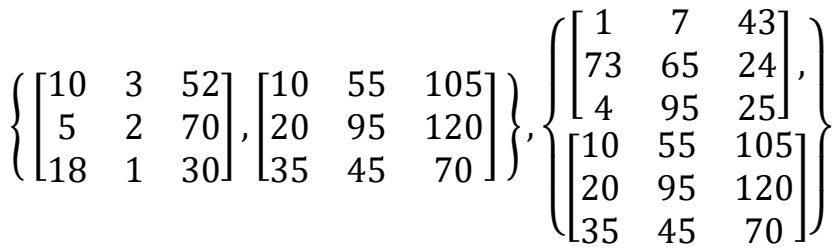

$,\left\{\left[\begin{array}{ccc}10 & 3 & 52 \\ 5 & 2 & 70 \\ 18 & 1 & 30\end{array}\right]\right\},\left\{\left[\begin{array}{ccc}1 & 7 & 43 \\ 73 & 65 & 24 \\ 4 & 95 & 25\end{array}\right]\right\}\left\{\left[\begin{array}{ccc}10 & 55 & 105 \\ 20 & 95 & 120 \\ 35 & 45 & 70\end{array}\right]\right\}$

There exists $\left\{\left[\begin{array}{ccc}10 & 3 & 52 \\ 5 & 2 & 70 \\ 18 & 1 & 30\end{array}\right],\left[\begin{array}{ccc}1 & 7 & 43 \\ 73 & 65 & 24 \\ 4 & 95 & 25\end{array}\right]\right\},\left\{\left[\begin{array}{ccc}10 & 55 & 105 \\ 20 & 95 & 120 \\ 35 & 45 & 70\end{array}\right]\right\}$ are fg-open sets

Such that: $\left\{\left[\begin{array}{ccc}10 & 3 & 52 \\ 5 & 2 & 70 \\ 18 & 1 & 30\end{array}\right],\left[\begin{array}{ccc}1 & 7 & 43 \\ 73 & 65 & 24 \\ 4 & 95 & 25\end{array}\right]\right\} \cap\left\{\left[\begin{array}{ccc}10 & 55 & 105 \\ 20 & 95 & 120 \\ 35 & 45 & 70\end{array}\right]\right\}=\varnothing$

And $\left\{\left[\begin{array}{ccc}10 & 3 & 52 \\ 5 & 2 & 70 \\ 18 & 1 & 30\end{array}\right],\left[\begin{array}{ccc}1 & 7 & 43 \\ 73 & 65 & 24 \\ 4 & 95 & 25\end{array}\right]\right\} \cup\left\{\left[\begin{array}{ccc}10 & 55 & 105 \\ 20 & 95 & 120 \\ 35 & 45 & 70\end{array}\right]\right\}=X$

Therefore $(\mathrm{X}, \mathcal{T})$ is fg-disconnecte space, but $(\mathrm{X}, \mathcal{T})$ is not disconnected space. 


\section{Theorem (2.13)}

Let $\mathrm{f}: \mathrm{X} \rightarrow \mathrm{Y}$ is fg-continuous, onto function. If $\mathrm{X}$ is fg-connected space, then $\mathrm{Y}$ is fg-connected space.

\section{Proof:}

Let $\mathrm{Y}$ is fg-disconnected space, thus there exists $\mathrm{H}, \mathrm{N}$ are disjoint non-empty open sets in $\mathrm{Y}$ such that $\mathrm{Y}=$ $\mathrm{H} \cup \mathrm{N}$. Since $\mathrm{f}$ is fg- continuous, onto function, thus $\mathrm{X}=\mathrm{f}^{-1}(\mathrm{H}) \cup \mathrm{f}^{-1}(\mathrm{~N})$, wheref $\mathrm{f}^{-1}(\mathrm{H})$ and $\mathrm{f}^{-1}(\mathrm{~N})$ is disjoint non empty and fg-open sets in $\mathrm{X}$. Thus, $\mathrm{X}$ is fg-disconnected space. This ambivalence the fact that $\mathrm{X}$ is fg-connected space; therefore, $\mathrm{Y}$ is fg- connected space.

\section{Proposition (2.14)}

If $\mathrm{X}$ is fg-connected space if and only if each fg-continuous function from $(\mathrm{X}, \mathcal{T})$ into the discrete two point $\{0,1\}$ is constant.

\section{Proof:}

$\Longrightarrow$ Let $X$ is fg-connected space and let $Y=\{0,1\}, \tau=\{\varnothing, Y,\{0\},\{1\}\}$

Suppose that there exist $\mathrm{f} \odot \mathrm{X}, \mathcal{T}) \rightarrow(\mathrm{Y}, \tau)$ such that $\mathrm{f}$ is fg-continuous function but $\mathrm{f}$ is not constant function. Then there exists $x, y$ in $X$ such that $f(x)=0, f(y)=1$. Since $f$ is fg-continuous function then $\mathrm{f}^{-1}(\{0\}), \mathrm{f}^{-1}(\{1\})$ are fg-open set in $\mathrm{X}$ but $\mathrm{f}^{-1}(\{0\})$ and $\mathrm{f}^{-1}(\{1\})$ are disjoint non-empty set and $\mathrm{X}=$ $\mathrm{f}^{-1}(\{0\}) \cup \mathrm{f}^{-1}(\{1\})$. Thus, $\mathrm{X}$ is fg-disconnected space; this contradicts the fact that $\mathrm{X}$ is fg-connected. Therefore, $\mathrm{f}$ is constant function.

$\Leftarrow$ Suppose every fg-continuous function from $(\mathrm{X}, \mathcal{T})$ into the discrete two points $\{0,1\}$ is constant.

Suppose that $(\mathrm{X}, \mathcal{T})$ is fg-disconnected space, then $\mathrm{F}, \mathrm{D}$ are fg-open set in $\mathrm{X}$ such that $F \neq \emptyset, D \neq \varnothing$ and $F \cap$ $D=\emptyset, F \cup D=\mathrm{X}$.

Let $\mathrm{f}:(\mathrm{X}, \mathcal{T}) \rightarrow(\mathrm{Y}, \tau)$ such that $(\mathrm{Y}, \tau)$ is fg-disconnected space, $\mathrm{Y}=\{0,1\}$

But $f(X)= \begin{cases}0 & \text { if } x \in F \\ 1 & \text { if } \quad x \in D\end{cases}$ 
Then $f^{-1}(\varnothing)=\emptyset, f^{-1}(Y)=X, f^{-1}(\{0\})=F, f^{-1}(\{1\})=D$

Thus $f$ is fg-continuous function but $f$ is not constant function, then contradiction, therefore $(\mathrm{X}, \mathcal{T})$ is fgconnected space.

Definition (2.15)

A collection $H$ of fg-open sets in $X$ is said to be fg-open cover of $K \subseteq X$ if $K \subseteq U\left\{U_{\alpha}: U_{\alpha} \in H\right\}$ holds.

Definition (2.16)

A space $\mathrm{X}$ is said to be fg-compact if every fg-open cover of $\mathrm{X}$ has a finite subcover.

\section{Example (2.17)}

Each finite subset of a space $\mathrm{X}$ is fg-compact.

\section{Definition (2.18)}

Let $\mathrm{F}$ is subset of a space $\mathrm{X}$ then $\mathrm{F}$ is said to be compactly-fg-closed set if for every fg-compact set $\mathrm{E}$ in $\mathrm{X}$, $\mathrm{F} \cap \mathrm{E}$ is fg-compact.

Example (2.19)

$$
\begin{aligned}
& \text { Let } X=\left\{\begin{aligned}
{\left[\begin{array}{ccc}
10 & 3 & 52 \\
5 & 2 & 70 \\
18 & 1 & 30
\end{array}\right],\left[\begin{array}{ccc}
1 & 7 & 43 \\
73 & 65 & 24 \\
4 & 95 & 25
\end{array}\right] } \\
,\left[\begin{array}{ccc}
10 & 55 & 105 \\
20 & 95 & 120 \\
35 & 45 & 70
\end{array}\right]
\end{aligned}\right\} \\
& \text { be set and let } \left.A=\left\{\begin{array}{ccc}
10 & 3 & 52 \\
5 & 2 & 70 \\
18 & 1 & 30
\end{array}\right]\right\} \text { be }
\end{aligned}
$$

set in $\mathrm{X}$ such that $(\mathrm{X}, \mathcal{T})$ is indiscrete space

$$
\text { Since } A \cap\left\{\left[\begin{array}{ccc}
10 & 3 & 52 \\
5 & 2 & 70 \\
18 & 1 & 30
\end{array}\right]\right\}=\left\{\left\{\left[\begin{array}{lll}
10 & 3 & 52 \\
5 & 2 & 70 \\
18 & 1 & 30
\end{array}\right]\right\}\right\} \text { is fg-compact set }
$$


$A \cap\left\{\left[\begin{array}{ccc}1 & 7 & 43 \\ 73 & 65 & 24 \\ 4 & 95 & 25\end{array}\right]\right\}=\varnothing$ is fg-compact set
$\left.A \cap\left\{\left[\begin{array}{ccc}10 & 55 & 105 \\ 20 & 95 & 120 \\ 35 & 45 & 70\end{array}\right]\right]\right\}=\varnothing$ is fg-compact set

$A \cap\left\{\left[\begin{array}{ccc}10 & 3 & 52 \\ 5 & 2 & 70 \\ 18 & 1 & 30\end{array}\right],\left[\begin{array}{ccc}1 & 7 & 43 \\ 73 & 65 & 24 \\ 4 & 95 & 25\end{array}\right]\right\}=\left\{\left[\begin{array}{ccc}10 & 3 & 52 \\ 5 & 2 & 70 \\ 18 & 1 & 30\end{array}\right]\right\}$ is fg-compact set

$A \cap\left\{\left[\begin{array}{ccc}10 & 3 & 52 \\ 5 & 2 & 70 \\ 18 & 1 & 30\end{array}\right],\left[\begin{array}{ccc}10 & 55 & 105 \\ 20 & 95 & 120 \\ 35 & 45 & 70\end{array}\right]\right\}$

$=\left\{\left[\begin{array}{ccc}10 & 3 & 52 \\ 5 & 2 & 70 \\ 18 & 1 & 30\end{array}\right]\right\}$ is fg-compact set $A \cap\left\{\left[\begin{array}{ccc}1 & 7 & 43 \\ 73 & 65 & 24 \\ 4 & 95 & 25\end{array}\right],\left[\begin{array}{ccc}10 & 55 & 105 \\ 20 & 95 & 120 \\ 35 & 45 & 70\end{array}\right]\right\}=\varnothing$ is fg-compact set.

$\left.A \cap X=\left\{\begin{array}{ccc}10 & 3 & 52 \\ 5 & 2 & 70 \\ 18 & 1 & 30\end{array}\right]\right\}$ is fg-compact set

Therefore, A is compactly-fg-closed set.

\section{Theorem (2.13)}

Let $\mathrm{f}: \mathrm{X} \rightarrow \mathrm{Y}$ is fg-continuous, onto function. If $\mathrm{X}$ is fg-connected space, then $\mathrm{Y}$ is fg-connected space.

\section{Proof:}

Let $\mathrm{Y}$ is fg-disconnected space, thus there exists $\mathrm{H}, \mathrm{N}$ are disjoint non empty open sets in $\mathrm{Y}$ such that $\mathrm{Y}=$ $\mathrm{H} \cup \mathrm{N}$. Since $\mathrm{f}$ is $\mathrm{fg}$ - continuous, onto function, thus $\mathrm{X}=\mathrm{f}^{-1}(\mathrm{H}) \cup \mathrm{f}^{-1}(\mathrm{~N})$, wheref $\mathrm{f}^{-1}(\mathrm{H})$ and $\mathrm{f}^{-1}(\mathrm{~N})$ are disjoint non empty and fg-open sets in $\mathrm{X}$. Thus, $\mathrm{X}$ is fg-disconnected space. This ambivalence the fact that $\mathrm{X}$ is fg-connected space, therefore $\mathrm{Y}$ is fg- connected space.

\section{Proposition (2.14)}

If $\mathrm{X}$ is fg-connected space if and only if each fg-continuous function from $(\mathrm{X}, \mathcal{T})$ into the discrete two points $\{0,1\}$ is constant. 


\section{Proof:}

$\Longrightarrow$ Let $X$ is fg-connected space and let $Y=\{0,1\}, \tau=\{\varnothing, Y,\{0\},\{1\}\}$

Suppose that there exist $\mathrm{f}:(\mathrm{X}, \mathcal{T}) \rightarrow(\mathrm{Y}, \tau)$ such that $\mathrm{f}$ is fg-continuous function but $\mathrm{f}$ is not constant function, then there exists $\mathrm{x}, \mathrm{y}$ in $\mathrm{X}$ such that $\mathrm{f}(\mathrm{x})=0, \mathrm{f}(\mathrm{y})=1$.

Since $\mathrm{f}$ is fg-continuous function then $\mathrm{f}^{-1}(\{0\}), \mathrm{f}^{-1}(\{1\})$ are fg-open set in $\mathrm{X}$ but $\mathrm{f}^{-1}(\{0\})$ and $\mathrm{f}^{-1}(\{1\})$ are disjoint non- empty set and $\mathrm{X}=\mathrm{f}^{-1}(\{0\}) \cup \mathrm{f}^{-1}(\{1\})$. Thus, $\mathrm{X}$ is fg-disconnected space; this contradicts the fact that $\mathrm{X}$ is fg-connected, therefore, $\mathrm{f}$ is constant function.

$\Leftarrow$ Suppose every fg-continuous function from $(\mathrm{X}, \mathcal{T})$ into the discrete two points $\{0,1\}$ is constant.

Suppose that $(\mathrm{X}, \mathcal{T})$ is fg-disconnected space, then F, D are fg-open set in $\mathrm{X}$ such that $F \neq \emptyset, D \neq \emptyset$ and $F \cap D=\emptyset, F \cup D=\mathrm{X}$

Let $\mathrm{f}:(\mathrm{X}, \mathcal{T}) \rightarrow(\mathrm{Y}, \tau)$ such that $(\mathrm{Y}, \tau)$ is fg-disconnected space, $\mathrm{Y}=\{0,1\}$

But $f(\mathrm{X})= \begin{cases}0 & \text { if } x \in F \\ 1 & \text { if } \quad x \in D\end{cases}$

Then $f^{-1}(\varnothing)=\emptyset, f^{-1}(\mathrm{Y})=\mathrm{X}, f^{-1}(\{0\})=F, f^{-1}(\{1\})=D$

Thus $f$ is fg-continuous function but $f$ is not constant function, then contradiction, therefore, $(\mathrm{X}, \mathcal{T})$ is fgconnected space.

Definition (2.15)

A collection $H$ of fg-open sets in $X$ is said to be fg-open cover of $K \subseteq X$ if $K \subseteq U\left\{U_{\propto}: U_{\alpha} \in H\right\}$ holds.

\section{Definition (2.16)}

A space $\mathrm{X}$ is said to be fg-compact if every fg-open cover of $\mathrm{X}$ has a finite subcover. 


\section{Example (2.17)}

Each finite subset of a space $\mathrm{X}$ is fg-compact.

Definition (2.18)

Let $F$ is subset of a space $X$ then $F$ is said to be compactly-fg-closed set if for every fg-compact set $E$ in $\mathrm{X}, \mathrm{F} \cap \mathrm{E}$ is fg-compact.

\section{Example (2.19)}

Let $X=\left\{\begin{array}{rrr}{\left[\begin{array}{ccc}10 & 3 & 52 \\ 5 & 2 & 70 \\ 18 & 1 & 30\end{array}\right],\left[\begin{array}{ccc}1 & 7 & 43 \\ 73 & 65 & 24 \\ 4 & 95 & 25\end{array}\right]} \\ ,\left[\begin{array}{rrr}10 & 55 & 105 \\ 20 & 95 & 120 \\ 35 & 45 & 70\end{array}\right]\end{array}\right\}$

be set and let $\left.A=\left\{\begin{array}{ccc}10 & 3 & 52 \\ 5 & 2 & 70 \\ 18 & 1 & 30\end{array}\right]\right\}$ be

set in $\mathrm{X}$ such that $(\mathrm{X}, \mathcal{T})$ is indiscrete space

Since $\left.A \cap\left\{\left[\begin{array}{ccc}10 & 3 & 52 \\ 5 & 2 & 70 \\ 18 & 1 & 30\end{array}\right]\right\}=\left\{\left[\begin{array}{lll}10 & 3 & 52 \\ 5 & 2 & 70 \\ 18 & 1 & 30\end{array}\right]\right\}\right\}$ is fg-compact set

$A \cap\left\{\left[\begin{array}{ccc}1 & 7 & 43 \\ 73 & 65 & 24 \\ 4 & 95 & 25\end{array}\right]\right\}=\varnothing$ is fg-compact set

$\left.A \cap\left\{\left[\begin{array}{ccc}10 & 55 & 105 \\ 20 & 95 & 120 \\ 35 & 45 & 70\end{array}\right]\right]\right\}=\varnothing$ is fg-compact set

$\left.A \cap\left\{\left[\begin{array}{ccc}10 & 3 & 52 \\ 5 & 2 & 70 \\ 18 & 1 & 30\end{array}\right],\left[\begin{array}{ccc}1 & 7 & 43 \\ 73 & 65 & 24 \\ 4 & 95 & 25\end{array}\right]\right\}=\left\{\begin{array}{ccc}10 & 3 & 52 \\ 5 & 2 & 70 \\ 18 & 1 & 30\end{array}\right]\right\}$ is fg-compact set

$A \cap\left\{\left[\begin{array}{ccc}10 & 3 & 52 \\ 5 & 2 & 70 \\ 18 & 1 & 30\end{array}\right],\left[\begin{array}{ccc}10 & 55 & 105 \\ 20 & 95 & 120 \\ 35 & 45 & 70\end{array}\right]\right\}$

$=\left\{\left[\begin{array}{ccc}10 & 3 & 52 \\ 5 & 2 & 70 \\ 18 & 1 & 30\end{array}\right]\right\}$ is fg-compact set $A \cap\left\{\left[\begin{array}{ccc}1 & 7 & 43 \\ 73 & 65 & 24 \\ 4 & 95 & 25\end{array}\right],\left[\begin{array}{ccc}10 & 55 & 105 \\ 20 & 95 & 120 \\ 35 & 45 & 70\end{array}\right]\right\}=\varnothing$ is fg-compact set.

$\left.A \cap X=\left\{\begin{array}{ccc}10 & 3 & 52 \\ 5 & 2 & 70 \\ 18 & 1 & 30\end{array}\right]\right\}$ is fg-compact set. Therefore, A is compactly-fg-closed set. 


\section{The Proposed Method}

This section will introduce the proposed method, which will deploy based on the concepts of Matrices. The research methodology will be experimental testing of image enhancement procedure proposed by Mohammed H. R. (Mohammed \& Hussain, 2021). We replaced the use of fuzzy set by using fg-disconnected space and compactly fgclosed set to determine the brightness and brightness of the internal components of the types of color images, gray and binary. We will consider the following:

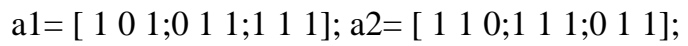

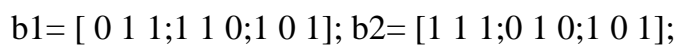

z1= imfilter(I,a); z2=I mfilter(I,b $;$ z3 = imfilter(I,x);

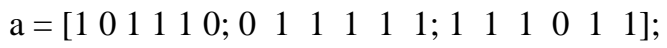

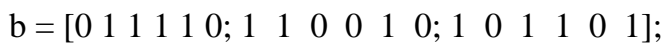

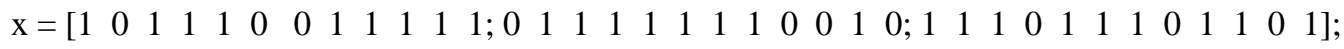

This work will focus on three types of images include (Color images, gray images, Binary images).

\section{Results and Discussion}

The section will discuss the implementation of proposed method and validate its results. The algorithm presented in Figure 1 is implemented to test the proposed method.

\section{Algorithm 1: fg-disconnected space algorithm}

Input: standardize image (Color, Gray)

Output: Enhanced Image with less information losses

Step 1: Select and process the Image Iteratively $(1.1-1.3)$ until enhanced image is achieved or the maximum number of iterations is reached. Then go to Step 2.

1.1 Implement the Matrixes filter Z1 and Z2, and choose the best result.

1.2 Delete regions to obtain image features using compactly-fg-closed set.

1.3 Convert the resulted image (1.2) to Binary format,

Step 2: Compared the results of selected images and their components by using matrices A, B, X.

Step 3: Compared the results of selected images Using histogram method to validate results. 
The proposed system is used samples of images from MATLAB library database with the three format (Color, Gray, and binary) as shown in Figure 2. Figure 3 represents the filter effect of matrix $Z_{1}$ and $Z_{2}$ on the selected images. Note that the illumination is to the hand of gray photos and this leads to jammed components and areas in the images.

Figure 4 shows converting images to binary format and delete components and regions to obtain color, gray and binary images using compactly-fg-closed set. Figure 5 shown performed process of deleting Large Regions for Color, gray and binary Images using fg-disconnected space.

Figure 5. results of deleting Large Regions for images Color, gray and binary utilizing fg-disconnected space. Figure 6 presents the output of three images that lost some properties from the original image according to matrices A, B, and $\mathrm{X}$ so that matrix A leads to the loss of less information than matrix $\mathrm{B}$ on the images, but matrix $\mathrm{X}$ leads to the loss of more information from the matrix $\mathrm{A}$ and $\mathrm{B}$ on the images.

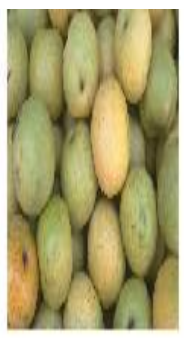

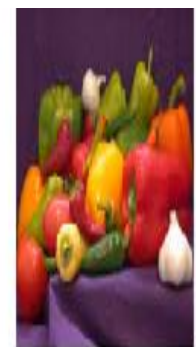
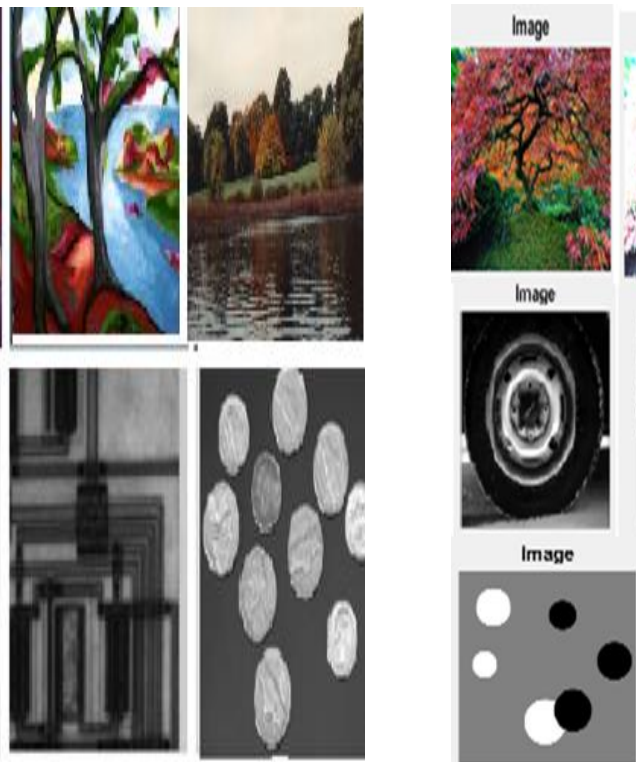

Figure 2. sample of images (Color, Gray).

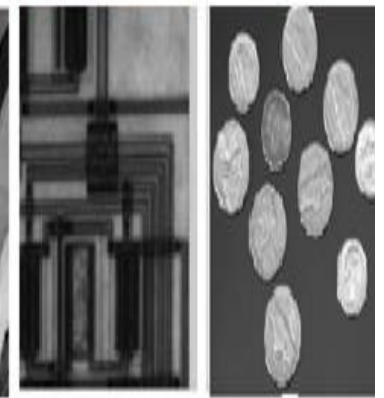

Figure 3. the filter effect on the matrixes $Z_{1}$ and $Z_{2}$. 


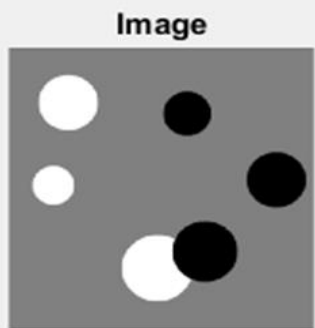

Image

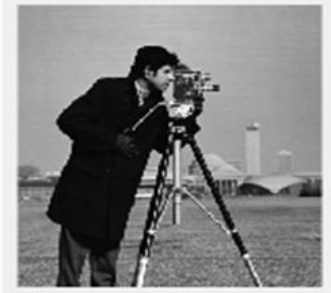

Image

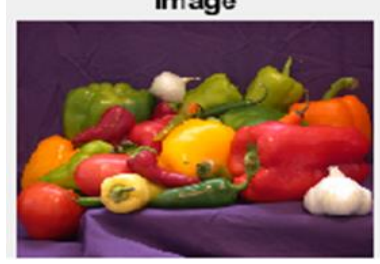

Binary Image

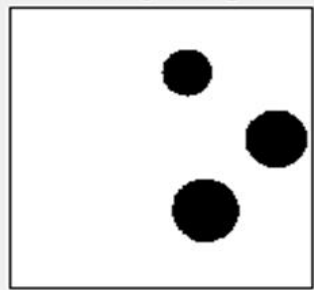

Binary Image

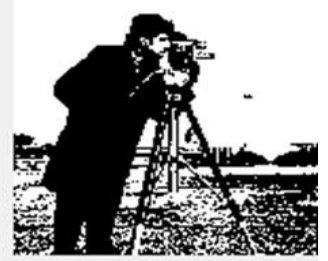

Binary Image

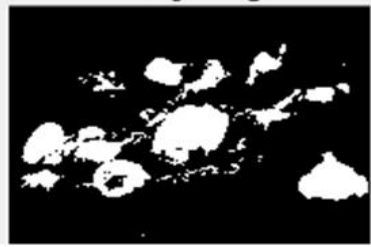

Figure 4. turned the pictures of whatever type into binary
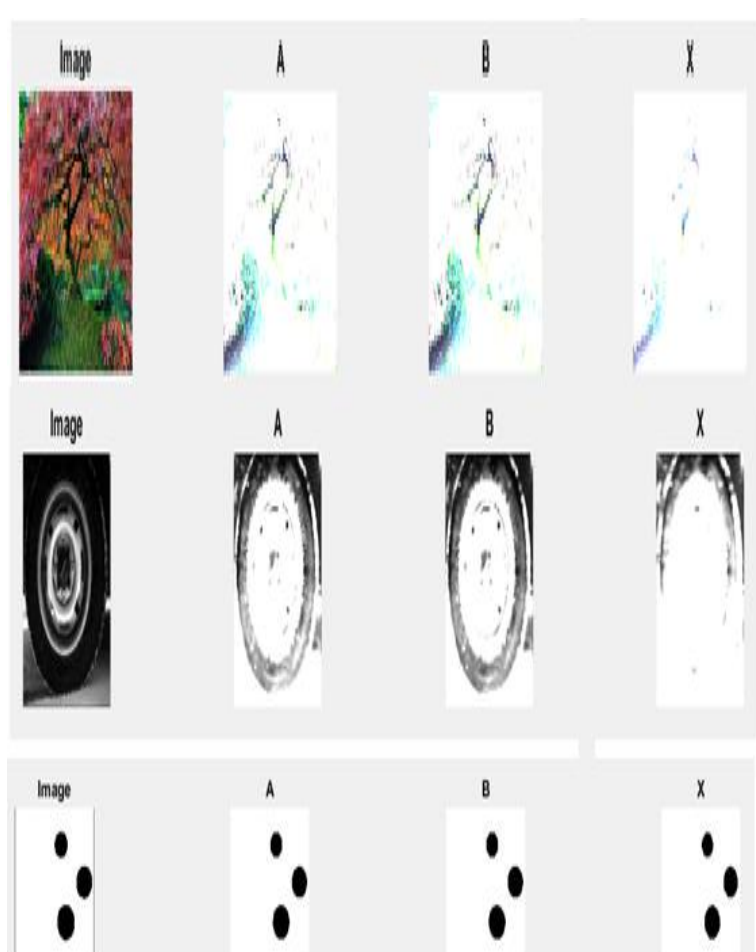

Figure 5. results of utilizing fg-disconnected space.
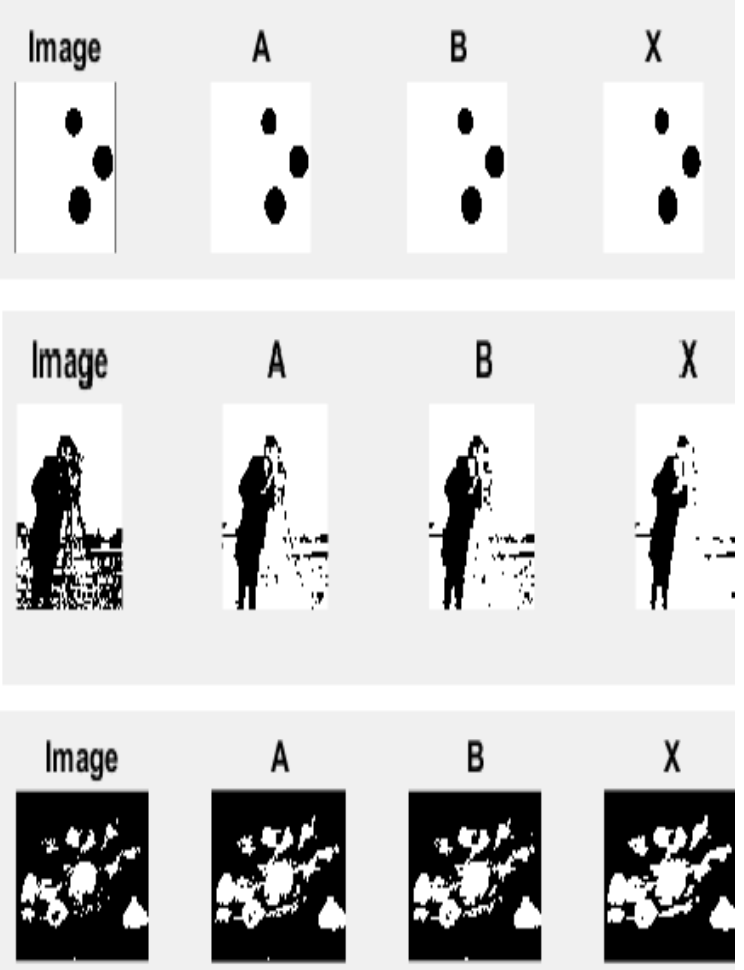

Figure 6. the selected images and their components by using matrices $\mathrm{A}, \mathrm{B}, \mathrm{X}$. 
The histogram method is approved that the resulted images are has the main features of the input image after using its suggested method with clearer and less noise as shown in Figure 7. Also, the suggested method reduced the needed time for image processing and enhancement.
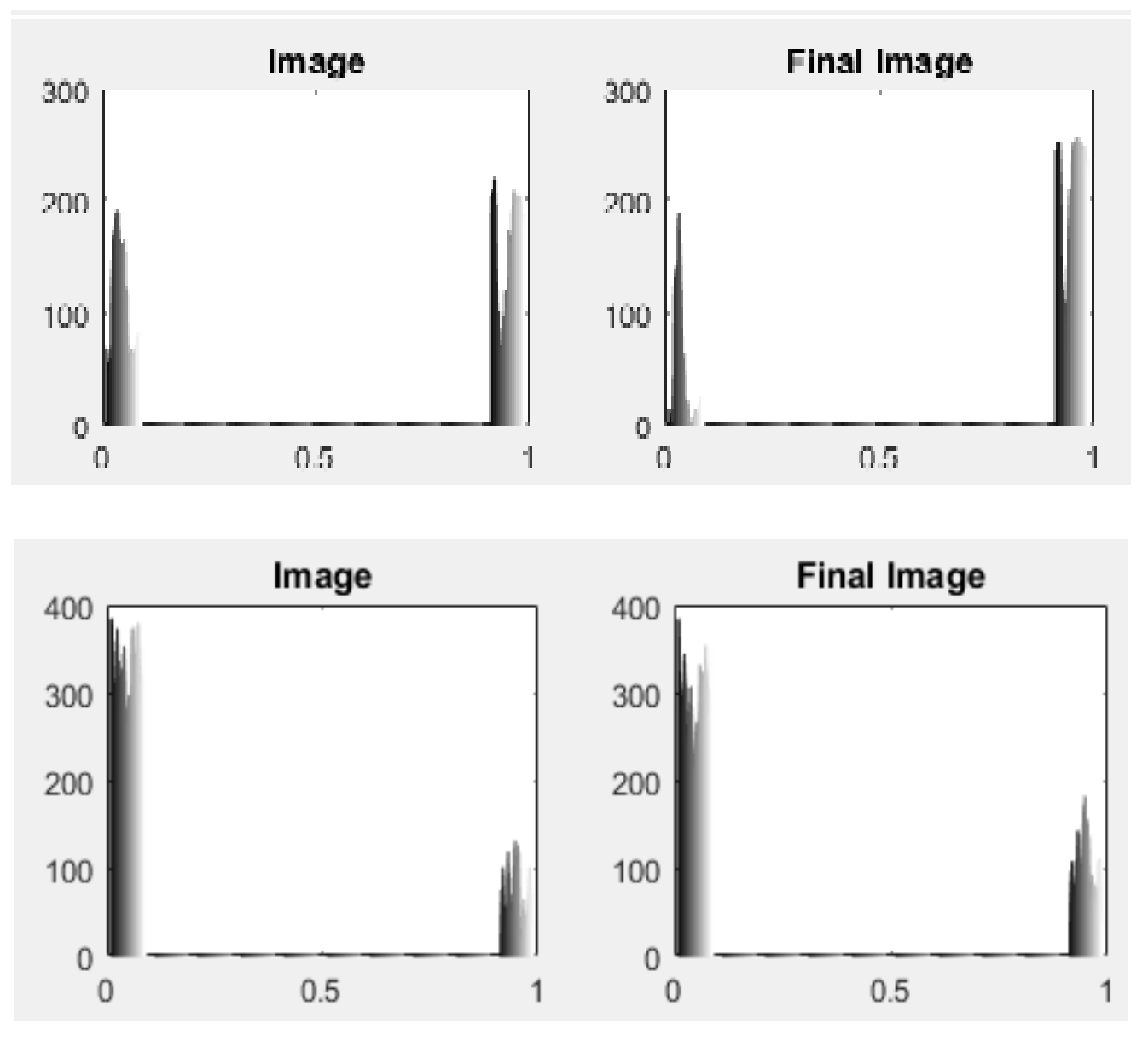

Figure 5. histogram for image and final image result

\section{Conclusion}

This work is used the Matrix filter based on compactly-fg-closed for enhancing the input image. The experiments are repeated several times using different images format (color and gray images) to get the needed results. The results indicated the following:

1.The lighting was very high in the color images but decreased in gray pictures and less in the binary images.

2. Data loss was grayed out more than binary the and finally colored.

3. The clarity of the objects was more in binary images than gray and finally colored.

4. The process of repeating the union and the intersection help to eliminate any deleted image components.

5. The use of fg-disconnected space leads to a white image, and the compactly-fg-closed set leads to a black image. 


\section{Acknowledgment}

The research leading to these results has no Research Project Grant Funding.

\section{References}

[1]. Al-Hatmi, M. O., \& Yousif, J. H. (2017). A review of Image Enhancement Systems and a case study of Salt \&pepper noise removing. International Journal of Computation and Applied Sciences (IJOCAAS), 2(3).

[2]. Al-Omeri, W., \& Jafari, S. (2019). On generalized closed sets and generalized pre-closed sets in neutrosophic topological spaces. Mathematics, 7(1), 1.

[3]. Banakh, T., \& Ravsky, A. (2020). On feebly compact paratopological groups. Topology and its Applications, $284,107363$.

[4]. Caldas, M., Georgiou, D. N., \& Jafari, S. (2003). Characterizations of low separation axioms via. Boletim da Sociedade Paranaense de Matemática, 21(1-2), pp 97-111.

[5]. Hassin, A., \& Abbood, D. (2021). Human-Ear Recognition Using Scale Invariant Feature Transform. Artificial Intelligence \& Robotics Development Journal, 1-12.

[6]. Ibraheem, D. (2008). On feebly generalized closed sets. Uruk Refereed Journal for Scientific Studies Issued by Al-Muthana University (1), pp131-139.

[7]. Levine, N. (1963). Semi-open sets and semi-continuity in topological spaces. The American mathematical monthly, 70(1), pp 36-41.

[8]. Levine, N. (1970). Generalized closed sets in topology. Rendiconti del Circolo Matematico di Palermo, 19(1), pp 89-96. Hasoon, F. N., Yousif, J. H., Hasson, N. N., \& Ramli, A. R. (2011). Image Enhancement Using Nonlinear Filtering Based Neural Network. Journal of Computing, 3(5), 171-176.

[9]. Mohammed, H. R., \& Hussain, Z. M. (2021). Hybrid Mamdani Fuzzy Rules and Convolutional Neural Networks for Analysis and Identification of Animal Images. Computation, 9(3), 35.

[10]. Navalagi, G. B. (2000). " Definition bank" in general topology. [ Accessed 20 April 2021] http://at.yorku.ca/i/d/e/b/75.htm

[11].Resan, D. I. (2011). Feebly Generalized Closed Mappings. Journal of Al-Qadisiyah for computer science and mathematics, 3(1), 94-100.

[12]. Shaaban, H. (2021). Enhanced Region of Interest Extraction method for Finger Vein Images. Artificial Intelligence \& Robotics Development Journal, 13-25.

\section{(c) (D) Author(s) and ACAA permit unrestricted use, distribution, and reproduction in any medium, provided the original work with proper citation. This work is licensed under Creative Commons Attribution International License (CC BY 4.0).}

